\title{
LA ECONOMETRÍA EN EL ECUADOR SUS ANTECEDENTES Y PRIMEROS PASOS
}

\section{ECONOMETRICS IN ECUADOR: BACKGROUND AND FIRST STEPS}

\section{OSWALDO DÁVILA ANDRADE ${ }^{1}$}

Recibido: 12 de abril

Aceptado: 1 de mayo

\footnotetext{
${ }^{1}$ El autor se graduó de economista en la Pontificia Universidad Católica del Ecuador en 1962. En 1965 obtuvo una maestría en Economía, con especialización en econometría, en la Universidad de Southern California, USC., Estados Unidos. En 1967, una vez cumplidos los requisitos obligatorios, incluida la aprobación de su tesis intitulada "Ensayos en econometría, aplicados a la realidad ecuatoriana", la PUCE le confirió el título de Doctor en Ciencias Económicas. Fue el primer doctorado en economía conferido por la Universidad.
} 



\title{
LA ECONOMETRÍA EN EL ECUADOR SUS ANTECEDENTES Y PRIMEROS PASOS
}

\section{ECONOMETRICS IN ECUADOR: BACKGROUND AND FIRST STEPS}

Oswaldo Dávila Andrade

Palabras clave: Historia, econometría, modelos económicos, planificación, crecimiento, desarrollo tecnológico

\begin{abstract}
Keywords: History, econometrics, economic models, planning, growth, technological development
\end{abstract}

\section{RESUMEN}

Este artículo repasa, a grandes rasgos, cómo nacieron, maduraron y se consolidaron algunos conceptos y definiciones teóricas fundamentales que se usan en una disciplina incorporada oficialmente al lenguaje científico, tales como econometría.

Cuenta de la experiencia del autor a principios de los 60 en el uso y los avatares de esta herramienta científica, en un país de escaso desarrollo como el Ecuador de entonces.

Relata como la planificación contribuyó a diseñar y aplicar modelos econométricos, útiles y sin pretensiones, compensando con esfuerzo humano e ingenio la carencia de hardware que sufrían los países periféricos frente a los centros hegemónicos de poder. 
La vertiginosa transformación tecnológica que ocurrió a fines del siglo XX es ilustrada con ejemplos actuales, los cuales llevan a que el autor termine el ejercicio expresando una mezcla de esperanza y temor hacia ese híper-tecnológico futuro que ya está aquí: la Revolución 5.0.

\section{SUMMARY}

This article intent to review, in broad terms, how some concepts and definitions, widely used in economic literature for more than a century, were born, matured, expanded and have been applied in practice. As it is econometrics.

The author describes his personal experience on how economic models were useful tools for economic and social planning, Also, shows how, if properly applied, econometric models can contribute to design long term growth policies and be used as testable blueprints for the road towards economic and social progress.

The paper also makes a brief analytical comment on the repercussions of the explosive evolution of technology and its beneficial but also scary effects if mishandled by the dominant corporations and governments.

It calls on the Academic Community to take its share of responsibility in shaping up the 5.0 Revolution.

\section{INTRODUCCIÓN}

En el siglo XX, hasta la década de los años 40 no existía en el lenguaje de economistas y sociólogos aquello de "subdesarrollo" y "desarrollo", tan comunes ahora en la literatura económica. Los países y sociedades, o eran pobres o eran ricos o estaban en proceso de evolución de la primera a la segunda categoría. Los estudiosos de la economía y algunos organismos internacionales, en general, asimilaban la pobreza con el re- traso y la riqueza con el progreso y así manejaban sus instrumentos de análisis de los problemas económicos y sociales.

Pero, con licencia de la historia, podría decirse que los términos fueron inventados por los traductores del libro que Joseph Schumpeter escribió en 1911, en alemán, Theorie der wirtschaftlichen Entwickllung y que en 1934 se tradujo al inglés como Theory of Economic Development. En 1941 se publicó en es- 
pañol con el título "Teoría del Desenvolvimiento Económico".

Desde entonces, la expresión "desarrollo económico" comienza a aparecer en escritos técnicos y documentos de la ONU; su legitimación final, al parecer se dio en el Punto Cuarto del discurso de posesión presidencial de Harry Truman, en 1949, en el cual se hace referencia a vastas regiones atrasadas de la Tierra como "mundo subdesarrollado" y propone "ayudarles a darse cuenta de sus aspiraciones para una mejor vida y en cooperación con otras naciones fomentar la inversión de capital en áreas necesitadas de desarrollo".

\section{EL DESARROLLO Y LOS MODELOS ECONÓMICOS}

Pasar del subdesarrollo al desarrollo ha sido el leitmotiv de varias generaciones de economistas y científicos sociales y la bibliografía sobre el tema es inmensa. Para presentar sus ideas y argumentos, muchos de ellos utilizaron fórmulas y expresiones matemáticas que talvez a partir de Quesnay y su $\mathbf{T a}$ bleau Economique, fueron los primeros intentos de esquematizar un sistema económico. Comenzaron a llamarse -y continúan llamándose- modelos económicos.

Un "modelo económico" puede definirse como un esquema mental, una representación, necesariamente simplificada, de un sistema económico: un país, una región, el mundo. Si esta represen- tación se la hace en términos matemáticos, con sustento estadístico, se obtiene un "modelo econométrico".

La "econometría", a su vez, es una disciplina científica que, desde principios del siglo XX, se ha ido conformando $y$ fortaleciendo para medir, interpretar, explicar y proyectar, a veces con demasiada pretensión, el comportamiento y las perspectivas de los diversos sucesos, fenómenos y dimensiones que conforman un sistema económico. Es el resultado de una evolución simbiótica de instrumentos teóricos y analíticos de dos ciencias formales, las matemáticas y la estadística y una ciencia empírica, fáctica, la economía. 


\section{LA ECONOMETRÍA Y LOS “MODELOS”}

El término 'econometría' fue utilizado por primera vez por Pawel Ciompa en1910; su aceptación formal por parte de los científicos sociales, la academia y la política se oficializa hacia 1930 y culmina con la creación, en Diciembre del mismo año, de la Econometric Society en Cleveland, Ohio. Más adelante, uno de los más destacados miembros fundadores de esta Sociedad, Ragnar Frisch, "oficializó" el bautizo del término en un artículo de 1936 titulado «Note on term 'Econometrics'», publicado en 1936 en la revista Econometrica $(4,95)$.

Su validez y prestigio como mecanismo de análisis e interpretación de la realidad económica y social ha ido evolucionando pari passu con la velocidad del desarrollo de la tecnología aplicada a la identificación, disponibilidad, volumen y procesamiento de los hechos económicos. La empatía entre informática, estadística y matemática ha sido un dinamo efectivo para el progreso y confiabilidad de los modelos econométricos.

Cabe advertir que los modelos pueden construirse y son aplicables a cualquier conjunto interrelacionado de la actividad humana: un país, una empresa, un sector (como la salud p. ej.), la desocupación, el desarrollo espacial, el medio ambiente. Casi no tienen límite, siempre que se cuente con la información y la capacidad técnica para procesarla. La CIA y la NASA en la carrera por el dominio espacial han utilizado desde hace tiempo, y con éxito, muy refinados modelos'.

\section{LA TEORÍA DEL DESARROLLO Y LOS MODELOS}

Los primeros modelos econométricos para propósitos de análisis económico, fueron desarrollados por autoridades como Wright $(1915,1928)$, Working (1927), Tinbergen (1930) y Frisch (1933). Estuvieron limitados a fenómenos específicos tales como el análisis de la demanda o los ciclos económicos. Estas aplicaciones fueron estableciendo y definiendo interrelaciones estructurales entre diferentes variables económicas, pero solo se aplicaron a las economías desarrolladas, que eran las únicas en capacidad de proveer, depurar y procesar,

\footnotetext{
${ }^{1}$ La carrera con los rusos para llegar a la Luna y la construcción del misil interconinental Minuteman requerían muy sofisticados sistemas de dirección, pero que cupieran dentro del diminuto cono de la punta de los proyectiles.
} 
en cantidad y calidad, el enorme volumen de datos que exigen los procesos estadísticos y las fórmulas matemáticas.

Por la primera mitad del siglo $X X$, en el mundo se dio una eclosión de científicos y académicos tratando de entenderlo, desenredarlo y ordenarlo, y formular teorías de comportamiento económico y social que contribuyeran al progreso o, por lo menos, explicaran el retraso y desigualdades imperantes.
Sin duda, la econometría les fue de gran ayuda para entender un mundo desquiciado que, no bien salió de una terrible guerra mundial en 1918, comenzó, de inmediato, por las sanciones y condiciones impuestas a Alemania, a crear todas las condiciones necesarias para que erupcione la Segunda Guerra (1939-1945), el conflicto internacional más cruento y desastroso que había conocido la humanidad².

\section{EL MARCO MUNDIAL}

Los años 40 y 50 fueron un período de grandes descubrimientos científicos y adelantos tecnológicos, impulsados en parte por las exigencias tecnológicas de la II Guerra (la alianza científico-militar) y de la reconstrucción posterior del Primer Mundo de esa época.

En el Tercer Mundo, en el mismo período, su funcionamiento y crecimiento económicos seguían dependiendo fundamentalmente de sus sectores primarios, en gran parte orientados a satisfacer la demanda externa, en un modelo mundial de intercambio que aún per- manecía sujeto a las reglas de juego neo colonialistas.

Pero el escenario de fondo, los actores y el argumento, estaban cambiando rápidamente.

Después de la I Guerra Mundial y de "los locos años 20" que le siguieron y que terminaron con la quiebra de Wall Street y principió la Gran Crisis en 1929, se produjo lo que podríamos llamar una revolución intelectual en el pensamiento económico de la época, el cual comenzó con la gran obra de John M. Keynes y cuyos postulados aún continúan vigentes.

\footnotetext{
${ }^{2}$ Despues de las reuniones de Yalta, Postman, en San Francisco, USA, la representación alemana calificó de "puñaladas por la espalda" a las onerosísimas condiciones impuestas por los Aliados.
} 


\section{AMÉRICA LATINA}

Para entonces, ya iba tomando forma en América Latina una nueva doctrina, una nueva escuela de pensamiento, que trataba de replantearse los problemas del subdesarrollo y encontrar soluciones diferentes a las que proponía el capitalismo colonialista imperante.

El principal exponente de una nueva tendencia desarrollista, que más adelante se denominó "estructuralismo", fue sin duda, Raúl Prebisch y, junto a él, una pléyade de economistas y científicos sociales latinoamericanos (como Celso Furtado, Osvaldo Sunkel, Maria Concepcion Tavares, Enzo Faleto, Helio Jaguaribe y muchos otros) cuya contribución teórica y práctica, reforzada luego con la creación de la Comisión Económica para Latin (CEPAL) y el Instituto Latinoamericano de Planificación Económica y Social (ILPES), fueron construyendo, cimentando y difundiendo una línea de pensamiento y política que llevó a la formulación de la "teoría de la dependencia", sintetizadas en la te- sis Prebisch-Singer, cuya aceptación con renuencia de algunos expertos y países introdujo los conceptos de "centro-periferia" y de "sustitución de importaciones" como fórmulas estratégicas de crecimiento y desarrollo.

Varios gobiernos y países latinoamericanos acogieron con entusiasmo lo que parecía ser un camino viable de ordenamiento y progreso de sus economías, asoladas por la expoliación de recursos naturales, crisis fiscal, déficit externo, desempleo e inequidad.

La forma de atacar y desenredar los laberintos nacionales emergió casi espontáneamente; se formularon tesis que, todavía, se mantenían dentro del esquema keynesiano: se necesitaba autoridad y una mayor y más eficiente presencia del estado para tratar de reordenar el mercado y formular planes y programas de mediano y largo plazo que tuvieran objetivos y políticas nacionales claros y medibles y un esquema de rutas posibles para alcanzar esos objetivos.

\section{LA PLANIFICACIÓN}

En este escenario, la planificación apareció como un imperativo y se comenzó a aplicar en casi todos los países. No fue, obviamente, del agrado de los grupos de poder que eran hasta entonces usufructuarios de ese desorden; pero la realidad les obligó a aceptar una verdad de sentido común, no siempre entendida: 
Que la planificación no es una ideología, sino una herramienta efectiva, en cualquier modelo político, para la generación, obtención y organización de recursos con el fin de obtener un objetivo determinado; en este caso el crecimiento y desarrollo integral y sostenible que se buscaba para tener un país distinto y mejor.

\section{LA PLANIFICACIÓN EN EL ECUADOR}

En un país tradicionalmente agrodependiente (aún no había aparecido el petróleo), inequitativo y con frecuentes vaivenes políticos, la necesidad de planificar se había hecho presente desde hacía tiempo. Ya en 1954 el Presidente Jose María Velasco Ibarra había creado la Junta Nacional de Planificación y Coordinación Económica (Junapla), no por iniciativa del Banco Central del Ecuador ni del Banco Internacional de Reconstrucción y Fomento (BIRF), como se ha afirmado, sino "por la maduración política de esta idea, de elevar la planificación a la categoría de herramienta fundamental de gobierno" situación que se había afirmado y consolidado a lo largo de años de eficiencia y dedicación al servicio del país. (Ver Moncayo, Patricio. "La planificación estatal en el interjuego entre desarrollo y democracia". Edit. Fraga, Quito, Abril 2017).

El país, y especialmente sus estamentos políticos, productivos y académicos, acogieron con entusiasmo la nueva corriente reformista latinoamericana y el Ecuador puede contarse, en la región, como uno de los pioneros en aplicar el concepto y las técnicas de planificación, para tratar de racionalizar e impulsar su economía en el mediano y largo plazo, mediante cambios deliberados en su estructura de producción y en la redistribución de prioridades para la asignación de recursos destinados a alcanzar un desarrollo más equilibrado y menos excluyente que el que se vivía entonces.

Convertir en realidad esta aspiración nacional no era fácil: se requería decisión política, primero, y luego una estructura institucional y recursos humanos, financieros y tecnológicos suficientes.

Inesperadamente, en 1963 se dio una coyuntura histórica favorable: el golpe militar que derrocó al Presidente Arosemena Monroy puso a la cabeza del gobierno a una Junta Militar, la cual optó por dar un giro al estilo tradicional de funcionamiento del estado ecuatoriano, convirtiéndolo en un estado desarrollista, cuya concepción, estructuración y puesta en marcha debían sujetarse a una planificación de largo alcance. 
En Ecuador se hizo uno de los primeros intentos de planificación a largo plazo, bajo la égida de Raúl Prebisch y la Comisión Económica de Naciones Unidas para América Latina y el Instituto Latinoamericano de Planificación Económica y Social ILPES, con la colaboración de varios organismos técnicos y financieros del mundo. La responsabilidad de dirección y coordinación de este esfuerzo se encargó a la Junta Nacional de Planificación y Coordinación Económica (Junapla).

Se iniciaron de inmediato los trabajos, basados en numerosos estudios que, sobre distintos temas del desarrollo, desde su creación en 1954, había venido realizando la Junapla; algunos de ellos ya estaban en trámite de estudio para financiamiento de varios organismos internacionales. Con el apoyo de la ONU, CEPAI-ILPES, BID, Banco Mundial, se acometió de inmediato la formidable tarea de diseñar y elaborar un Plan Nacional de Desarrollo con un horizonte de diez años, el Plan 3000, como de inmediato lo bautizó el ingenio popular, por el número de páginas del documento que lo contenía.

Después de numerosas e intensas reuniones técnicas con delegados y representantes de todos los estamentos económicos y sociales del país, que argumentaban en favor y en contra de la inclusión en el Plan de algunos sectores y actividades (algunas de las cuales res- pondían a intereses específicos y cuestionables), se acordó que, como punto de partida, se necesitaba diseñar un marco de referencia agregado, cuantificable, comprobable, que fuera más allá del enunciado verbal y escrito de aspiraciones y objetivos para el futuro, es decir,

\section{un modelo global para el desarrollo del país.}

Nuevamente, numerosas reuniones y debates entre todos los actores de la sociedad ecuatoriana Ilevaron a definir los primeros esquemas de lo que sería un Plan de Desarrollo a Largo Plazo. Era una experiencia nueva y apasionante no solo para el país, sino que lo fue para América Latina. Se produjeron decenas de estudios, análisis, opiniones, todos los cuales necesitaban alguna forma de cuantificación coherente, realista y, sobre todo, periódicamente comprobable.

La respuesta se buscó y encontró por el lado técnicamente más apropiado y lógico: la econometría. Se optó por diseñar y construir un modelo matemático que incluyera las variables macroeconómicas esenciales para tener un marco global de crecimiento que, partiendo de la estructura económica y social vigente al año 1962, permitiera un ejercicio razonable y pragmático de proyecciones que llevaran a cumplir con los objetivos de desarrollo planteados por la sociedad y el Estado y coordinados técnicamente por la Junapla. 


\section{LA ECONOMETRÍA EN EL ECUADOR}

Las primeras incursiones del país en esta disciplina científica complicada como es la econometría, cumplieron a la fecha más de medio siglo. Hubo ejercicios, realizados especialmente por técnicos del Banco Central y del Ministerio de Fomento dirigidos por el Ingeniero José Corsino Cárdenas y sobre todo, por las aplicaciones didácticas de estadística matemática dirigidas por el Dr. Héctor Correa Pólit en las Universidades Central y Católica. Durante un tiempo fue una especie de disciplina esotérica, para iniciados.

Actualmente, es una herramienta casi indispensable para los economistas y científicos sociales, sustentada en los adelantos fabulosos de la tecnología. Se optó entonces por diseñar un modelo econométrico que incluyera las variables macroeconómicas esenciales para tener ese marco global de crecimiento y estructura.

No se tenían experiencias nacionales anteriores, pero había que construirlo, aun sabiendo, o presintiendo, las dificultades por venir, algunas casi insalvables, como sintéticamente se verá luego, con una sola consigna: Hacer lo mejor que se pueda, con lo que se tenga.

\section{EL MODELO, LA TECNOLOGÍA, LA LEY DE MOORE}

Para decidir sobre la formulación matemática de un modelo de crecimiento adecuado para un país de las características del Ecuador, fueron necesarias varias reuniones de expertos en distintas materias relevantes. Participaron calificados profesionales de CEPAL e ILPES y se contó, con el apoyo del PNUD, con la invalorable contribución y supervisión del Doctor Gerhard Tintner ${ }^{3}$. La contraparte ecuatoriana se conformó con los profe- sionales del Departamento de Programación Global de la Junapla, a esas fechas dirigido por el autor del presente trabajo.

Diseñado el marco teórico, el tipo de modelo escogido fue resultado de un análisis muy exigente de la calidad y cantidad de la información disponible y de la capacidad de procesamiento disponible en el pais.

No eran precisamente las ideales. Como casi en todos países del Tercer

\footnotetext{
${ }^{3}$ Sabio y reputado economista austriaco, autor de varios textos especializados en econometría y profesor en universidades europeas y norteamericanas; a la sazón, en USC, Los Angeles, California.
} 
Mundo, los recursos que se dedicaban para investigación habían sido históricamente, muy limitados, y los equipos de procesamiento disponibles (hardware), tenían décadas de atraso 4 .

\section{EL MODELO}

Para construir el modelo econométrico, se examinaron previamente y a fondo — casi se desmenuzaron - las posibilidades y limitaciones que ofrecería la información disponible en el Banco Central, la Superintendencia de Bancos, el INEC y muchas fuentes privadas, y los medios con que se contaba para procesarla. Se necesitaba diseñar y aplicar una expresión matemática significativa que fuera la referencia para las metas y las funciones de producción sectoriales, las cuales se calcularían utilizando la matriz de insumo-producto que estaba en elaboración.

Se pensó inicialmente en construir un Modelo de Máxima Verosimilitud con ecuaciones simultáneas como el desarrollado por Haavelmo y la Comisión Cowles, apoyados por la Universidad de Chicago, en 1952, pero ni la información era suficiente ni se tenían las facilidades computacionales necesarias.

Se recordó que la mayor restricción en la aplicación del modelo MVIC fue la falta de poder de la computación, lo cual esta gráficamente representado en las fotografías existentes del laboratorio de computación de la Comisión Cowles en 1952, cuando las computadoras no eran máquinas, sino personas que pasaban todo el día colocando números en las máquinas calculadoras de escritorio. Situación idéntica a la que la Junapla estaba enfrentada en 1962.

Este fue el problema computacional que llevó a la Comisión Cowles a desarrollar la alternativa más fácilmente computable del Modelo de Máxima Verosimilitud de Información Limitada (MVIL) y, en 1953, a Henry Theil a presentar el Modelo de Mínimos Cuadrados en dos etapas (MC2E).

Se pensó en esta última opción, pero luego, gracias a la experiencia y por sugerencia de nuestro asesor principal, se decidió que, a pesar de que los MC2E ofrecían una técnica que podría ser implementada no obstante los problemas

\footnotetext{
${ }_{4}^{4}$ Por ejemplo, la matriz de insumo-producto tuvo que ser invertida con calculadoras eléctricas Friden y con muchisima "mano de obra", cuando en los países ricos se trabajaba desde hacía tiempo con computadores de alta velocidad.
} 
computacionales, los Mínimos Cuadrados Ordinarios (MCO) eran -y continúan siéndolo hasta hoy - el método de estimación más ampliamente utilizado. Se decidió seguir este camino.

Finalmente, el grupo encargado de la modelización, bajo la dirección del Profesor Tintner, acordó utilizar como pivote central para el modelo, la función

\section{de producción Cobb-Douglas ${ }^{5}$.}

Como se sabe, esta formulación es un enfoque neoclásico que sirve para estimar la producción agregada de un país (o sector en ciertos casos) como una función del trabajo y el stock de capital, y permite estimar y medir los efectos de los cambios tecnológicos.

Los resultados -que pueden estimarse mediante la aplicación de formas logarítmicas para linealizar las funciones - son y pueden aplicarse como elasticidades. Esto permite hacer proyecciones sobre posibles tasas de crecimiento de la producción y del PIB y simular escenarios de referencia para introducirlos coherentemente en los modelos sectoriales.

La fórmula básica creada por
Cobb y Douglas y utilizada para el modelo ecuatoriano, con las adaptaciones necesarias, es la siguiente:

\section{(Ver Modelo Econométrico}

para Ecuador. Anexo 1).

$$
Y=A K^{\alpha} L^{\beta}
$$

\section{Donde:}

$\mathrm{Y}=$ Producción

A= Progreso tecnológico (exógeno), llamado también Productividad total de los factores (PTF)

$\mathrm{K}=$ Stock de capital

$\mathrm{L}=$ Número de trabajadores

a y $\beta=$ parámetros que representan el peso de los factores $(K$ y L ) en el PIB. Los parámetros varían entre 0 y 1 .

Nota importante: Más adelante, como el capital humano es considerado un factor de producción muy importante, en los estudios de Uzawa (1965) y Lucas (1988), se sustituyó el factor trabajo (L) por el factor capital humano (H). Se mantuvo la tecnología (A) y el capital financiero $(k)^{6}$.

\footnotetext{
${ }^{5}$ Dos célebres economistas, Charles W. Cobb y Paul H. Douglas, se plantearon en su época un teoría de la producción, ("A Theory of Production", The American Economic Review, vol.18, n.-1, Marzo 1928, pp. 139165). Los datos con los que se sustenta el modelo comprenden los años 1899-1922. A pesar del tiempo transcurrido y los cambios de estructuras económicas, el modelo mantiene su validez hasta la presente y es aún una herramienta útil para pronósticos del crecimiento económico.

${ }^{6}$ Dependiendo del sector, los efectos de los adelantos tecnológicos pueden ser muy grandes y, por tanto, afectar la ecuación global. (Ver Ley de Moore ...p.10).
} 
Los resultados - que se estiman mediante la aplicación del método de mínimos cuadrados ordinarios y de formas logarítmicas para linealizar las funciones - resultan ser, en rigor, elasticidades. Esto permite hacer proyecciones sobre posibles tasas de crecimiento de la producción y del PIB y simular escenarios de referencia para introducirlos coherentemente en los modelos sectoriales.

La ordenada al origen en la ecuación de las funciones de producción puede ser construida tambien a partir de funciones de producción sectoriales, y modificada según los resultados que se obtenga en estas últimas.

\section{LA TECNOLOGÍA}

El atraso ecológico en el país limitó mucho las aspiraciones del equipo técnico que trabajaba con el modelo. Hay que recordar que, hasta mediados de los años 50, el procesamiento de datos lo hacían computadoras con potencia generada a base de válvulas electrónicas, las cuales fueron sustituidas por semiconductores llamados transistores (acrónimo de su denominación en inglés).

El primer gran salto hacia lograr que las operaciones se hagan a las velocidades astronómicas que vemos ahora, se dio entre fines de los 50 y principios de los 60, cuando se pasa de utilizar la potencia de los transistores a la potencia que comienzan a generar los circuitos integrados en chips y luego microchips; ingenios estos últimos que surgieron en 1962, en buena parte por la presión del Departamento de Defensa de los Estados Unidos.
Si se suman estas mejoras a las que se incorporaron luego, gradual pero rápidamente, a las unidades de memoria, los sistemas de redes, el software y los sensores, nos encontramos con una capacidad total de procesamiento que crece exponencialmente, con resultados cuantitativos casi inimaginables.

Pero, para países del Tercer Mundo esta tecnología - software y hardware - no estaba disponible. En el Ecuador, el modelo global, la matriz de insumo-producto y los modelos sectoriales que se fueron aplicando en la estructuración del Plan, se vieron seriamente limitados por la velocidad de procesamiento y, por supuesto, fueron cuestionados y criticados, con razón y sin ella, No obstante, sirvieron para dibujar horizontes razonables de optimismo en un país pequeño, pero que contaba con variados y envidia- 
bles recursos humanos y materiales que había que usarlos racional y eficientemente.
Esos horizontes aún no estaban distorsionados por los espejismos petroleros.

\section{LA “LEY DE MOORE”}

La tecnología afecta e influye en todos los órdenes de actividad que puede imaginar el cerebro humano. Las ciencias no son sino una fracción infinitesimal del conocimiento del universo. Como lo es la física; o la economía.

Para percibir el poder, los efectos y la magnitud de los adelantos tecnológicos y las restricciones que se enfrentan en todos los ámbitos del saber, parece útil, aunque sea mencionar, cómo y cuánto ha avanzado la humanidad en campo.

Parece que el ingeniero cofundador de Intel, Gordon E. Moore, fue el primero en darse cuenta del fenomenal impacto que la tecnología estaba teniendo y con qué fuerza se proyectaba hacia el futuro. Enunció, el 19 de Abril de 1965, una ley empírica (o un teorema, si se quiere) que hoy se la conoce como Ley de Moore la cual, en términos simples, expresa: "Aproximadamente cada dos años se duplica el número de transistores en un microprocesador". Esto significa que la industria de los chips está duplicando producción de potencia cada dos años. Moore afirmó que esto ocurriría al menos durante veinte años. Si esta ley se siguiera cumpliendo — algo que los expertos consideran improbable- la capacidad de generar energía sería infinita. (Ver gráfico. Anexo 2).

No ha sido posible acceder a información reciente sobre este tema. No obstante, para graficar de alguna manera lo que significa duplicar potencia cada dos años hay que mirar solo un par de cifras: en 26 años, el número de transistores en un chip se ha incrementado 3200 veces $y$, actualmente, esta ley se aplica, por ejemplo, a ordenadores personales y teléfonos móviles o celulares, cuya proliferación sobrepasa, de lejos, la población mundial.

Cuando Moore hizo sus declaraciones, el chip más complejo contaba con 64 transistores; hoy, un chip Pentium IV tiene $\mathbf{5 5}$ millones de transistores! $Y$ la investigación nanodimensional sigue su marcha imparable a una velocidad exponencial que los seres humanos comunes aún no estamos en capacidad de asimilar. Por ejemplo la Iniciativa de Informática Estratégica Acelerada (ASCI), creada en 1996 por el gobierno de Esta- 
dos Unidos, ha desarrollado una supercomputadora capaz de procesar más de un teraflop ${ }^{7}$.

Al momento, además, los técnicos han desarrollado nuevos materiales, más livianos que el silicio que se viene usando para construir los transistores. Estos nuevos materiales permiten inducir mucho más velocidad al flujo de ida y vuelta de los electrones, al costo más bajo y en el espacio más pequeño posible.

Y según los expertos, todavía se está lejos de llegar al límite....si existe.

Los rapidísimos avances tecnológicos son autoimpulsados por su propio dinamismo — como lo ha probado la Ley de Moore- y estimulados por las exigencias de un patrón de demanda que con las técnicas actuales de marketing parecen funcionar bajo los parámetros de la Ley de Say (1803) —la oferta crea su propia demanda-. Pero, además, esos avances y sus autores han sido y son constantemente presionados - por instituciones, gobiernos y empresaspara que desarrollen los hipersofistica- dos implementos que se necesitan en la multibillonaria batalla por el poder que sostiene desembozadamente la tríada de titanes Estados Unidos, Rusia y China ${ }^{8}$. Sus máximas dirigencias pugnan por ocupar el Trono de Júpiter o la Presidencia de la Tierra, como se quiera llamar, sin que importe para nada el costo humano, ecológico y cultural que conllevan la codicia y la soberbia desenfrenadas que, embadurnadas con la corrupción y la droga, campean a sus anchas por todo el globo terráqueo.

Sin duda, la humanidad está en un punto de inflexión (¿hacia arriba o hacia abajo?) rumbo a un mundo muy diferente del que hemos vivido hasta hoy.

Quizá quepa una reflexión final, que talvez merezca la atención de la Academia: Hasta hoy, la tecnología y sus impulsores nos han enseñado a amar lo que ellos aman producir; talvez es tiempo de que los seres comunes y corrientes les enseñemos a producir lo que nosotros amamos.

\footnotetext{
${ }^{7}$ Un flop es una operación de cálculo muy complicada —más que una operación de cálculo integral_que incluye decimales. A nivel informático, un teraflop es una operación que incluye UN BILLÓN de estos cálculos POR SEGUNDO!

8 ((En su Anuario 2018, el Stockholm International Peace Research Institute - SIPRI establece que el gasto militar mundial en 2017 fue de 1,739 billones de dólares (1 739000000000 000)).
} 


\section{REFERENCIAS BIBLIOGRÁFICAS}

Cobb Charles W. y Douglas Paul H. "A Theory of Production" (The American Economic Review"): "Un esquema completo para computar todas las elasticidades de demanda directay cruzada en un modelo con muchos sectores", 1959, Econometrica, Vol. 18, No. 1, marzo, 1928.

Friedman, Thomas L. "Gracias por llegar tarde". DEUSTO, Grupo Planeta, Colombia 2018.

Frisch, Ragnar. "Un esquema completo para computar todas las elasticidades de demanda directa y cruzada en un modelo con muchos sectores", 1959, Econometrica.

Frisch Ragnar "Nota sobre el Término'Econometría", 1936, Econometrica.

Frisch, Ragnar "Un esquema completo para computar todas las elasticidades de demanda directa y cruzada en un modelo con muchos sectores", 1959, Econometrica.

Haavelmo Trygve at the Cowles Commission. Article in Econometric-
Theory

31(01):84 - February2014. DOI: 10.1017/ S026646661400019

Moncayo, Patricio."La planificación estatal en el interjuego entre desarrollo y democracia". Flacso, Ecuador, 2017.

Salgado, Germánico, Edit. "25 años de planificación", Junta Nacional de Planificación, Quito, Ecuador, 1979.

Tinbergen, Jan. Econometrics. https:// www.jstor.org/stable/2226757

Tintner Gerhard. "Methodology of mathematical economics and econometrics" Chicago : University of Chicago Press, 1968. $113 \mathrm{p}$.

Tintner, Gerard, Econometrics. (London: Allen and Un-win, 1951. Pp. xii).

Tintner, Gerhrd \& Dávila Oswaldo, " Funciones de Producción para la Economía Ecuatoriana"- Junta Nacional de Planificación, Quito, Ecuador.1962 Principio del formulario 


\section{ANEXOS}

\section{ANEXO 1. UN MODELO ECONOMÉTRICO PARA ECUADOR}

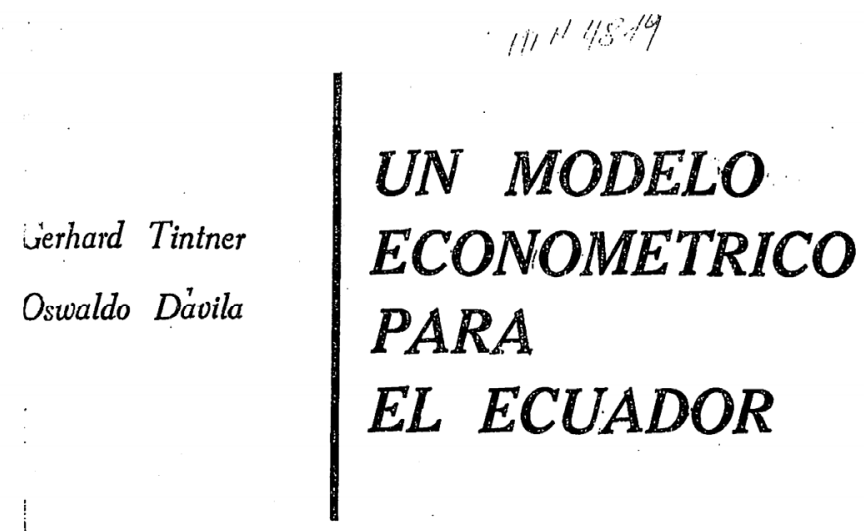

JUNTA NACIONAL DE PLANIFICACION Y COORDINACION ECONOMICA QUITO - ECUADOR 


\title{
UN MODELO ECONOMETRICO PARA EL ECUADOR
}

\author{
Gerhard Tintner Oswaldo Dávila
}

1. Un modelo keynesiano

Para la planificación se necesita construir modelos econométricos. Pero, en los palses subdesarrollados muchas veces faltan los datos necesarios para su construccion. Hemos construido un peque กิo modelo para el Ecuador, con base en los datos publicados por el Ban co Central del Ecuador. (*)

Nuestro modelo, muy modesto, contiene solamente cin co ecuaciones. $D_{e}$ éstas, solamente dos son ecuaciones que expresan el comportamiento de la economia ecuatoriana. Las otras tres son bási camente definiciones.

\section{Nuestras variables son:}

a)

Variables endogenas: Variables económicas cuyos valo res son determinados por la interacción de nuestras e cuaciones. Su número es igual al namero de ecuaciones del model o:

C - consumo personal nominal

$\mathrm{Y}=$ Producto Interno Bruto nominal

$\mathrm{P}=$ Indice de precios para el ingreso nacional

$\mathrm{X}=$ Producto Interno Bruto real

$D=$ empleo

(*) Memoria del Gerente 1961 
$-2-$

b) Variables exogenas: Estas Variables, por definicion, influyen en los valores de nuestras variables economicas end6genas, pero no son influenciadas por ellas:

$\mathrm{N}=$ poblacion

G . consumo páblico nominal

$I=$ inversiones brutas nominales

$E \quad$ exportaciones

M = importaciones

W - salario nominal por año para cada trabajador

La primera ecuación es una función de consumo; y es una relación lineal entre el consumo real per cápita (C/NP) y el ingreso real per cápita ( Y/.NP):

(1) $\mathrm{C} / \mathrm{NP}=\mathrm{a}+\mathrm{b}(\mathrm{Y} / \mathrm{NP})$

Designamos por $H=G+E-M$ una nueva variable exogena sumaria. La definición del PIB nominal es, entonces:

(2) $: Y=C+I+H$

El P.I. B real es, por definicion:

(3) $X \cong Y / P$

La demanda de mano de obra se deduce de la teorla de la pro ductividad marginal del empleo:

(4) $\quad \mathrm{dX} / \mathrm{dD}=\mathrm{W} / \mathrm{P}$

La última ecuación de nuestro sistema es una función de produccion, del tipo Cobb-Douglas (lineal en logaritmos) con un solo factor de produccion: empleo:

(5) $\log X=d+f \log D$ 
Es necesario hacer notar que el sistema es estático; no entran factores dinámicos como el tiempo $(t)$ o retrasos. Más adelante transformaremos este sistema en uno dinámico para planificación de largo plazo.

2.

\section{Estimación de los parámetros}

Si nosotros tenemos $N$ observaciones $(t=1,2 \ldots N)$ de todas las variables podemos estimar la relacion. (1), función del consumo, por una modificación del método de ecuaciones simultáneas. Si consideramos la cantidad $(\mathrm{I}+\mathrm{H}) / \mathrm{NP}$ como una variable exógena, las ecuaciones en la forma reducida son:

(6) $\quad \mathrm{Y} / \mathrm{NP}=\mathrm{K}_{1}+\mathrm{L}_{1}(\mathrm{I}+\mathrm{H}) / \mathrm{NP}=140,2600+$ $3,6798(\mathrm{I}+\mathrm{H}) / \mathrm{NP}$

(7) $\mathrm{C} / \mathrm{NP}=\mathrm{k}_{2}+\mathrm{L}_{2} \quad(\mathrm{I}+\mathrm{H}) / \mathrm{NP}=-140,2600+$ $2,6798(\mathrm{I}+\mathrm{H}) / \mathrm{NP}$

Estas dos ecuaciones (6) y (7) son estimadas con ayu da del método de minimos cuadrados.

Los parámetros de la función de consumo (1) pueden ser estimados como:
(8) $\quad a=\left(k_{1} L_{2}-L_{1} k_{2}\right) / L_{2}=497,2667$
(9) $\quad \mathrm{b}=\mathrm{L}_{1} / \mathrm{L}_{2}=0,5699$

Los parámetros de la función de producción (5) son esti mados por un metudo descrito por Klein: 
(10) $\log f=\sum[\log (W / P)-\log (X / D)] / N=0,\left[\begin{array}{l}{[i, 614707} \\ \text { (11) } d\end{array}\right]=\sum[\log X-f \log D] / N=7,51141$

Así tenemos para la función del consumo:

(12) $\mathrm{C}=497,2667 \mathrm{NP}+0,5699 \mathrm{Y}$

Su interpretacion es: Si, ceteris paribus, el Produc to real per cápita ( $\mathrm{Y} / \mathrm{NP})$ aumenta en un sucre, el consumo real per Cápila (C/NP) aumentará en 0,57 sucres, aproximadamente.

Como estimación de la función de la producción se tie ne:

$$
\text { (13) } \log \mathrm{x}_{\mathrm{t}}=7,51141+0,4119 \log \mathrm{D}_{\mathrm{t}}
$$

Esta relacion debe ser interpretada: si ceteris pari .. bus, el empleo aumenta en $1 \%$, el producto real en Ecuador aumentará en $2 / 5 \%$ aproximadamente.

3.

\section{Un modelo dinámico}

Es posible utilizar este modelo estático para predic ciones a corta plazo. Pero como se puede observar, la función de pro duccion depende solamente del empleo, y la hipotesis es que el capi tal es constante.

Esta no es una hipotesis muy real, especialmente en paises como el Ecuador que necesitan acumulación de capital. Una relación muy simple para la formación del capital (K) es : 
(14) $k_{t+1}=A K_{t}+I_{t}$

En Ecuador, la constante A (factor de depreciacion) es estimado como: $(1-0.0286)=0.9714$

La formula para el capital nominal en el afio $t$ es:

$\mathrm{K}_{t}=A^{t} \mathrm{~K}_{0}+A^{t-1} \mathrm{I}_{0}+A^{t-2} \mathrm{I}_{1}+\ldots \mathrm{I}_{t-1}$

Si introducimos una inversion media I en cada ađo, tenemos:

(16) $K_{t} \cdot A^{t} K_{0}+I\left(1-A^{t}\right) /(1-A)$

Pero, si $t$ es muy grande, y ya que $O<A<1$, en el 1 I mite: $t \rightarrow \infty ; y:$

(17) $K_{t}^{*}=I /(1-A)$.

En esta formula, I es la inversion media.

Es muy difícil estimar la influencia del capital en la economia ecuatoriana. Pero, en investigaciones anteriores hemos obteni do casi siempre $f+g$ - 1 ; si definimos por $g$ - la elasticidad de la produccion respecto del capital, se tiene:

$$
g=1-f=0,5881
$$

Su interpretacion es: si ceteris paribus el stock de capi tal aumenta en $1 \%$, el producto aumentará en $0,6 \%$ aproximadamente.

La nueva función de producción es:

(19) $\log \mathrm{X}=\mathrm{d}^{\prime}+\mathrm{P} \log \mathrm{D}+\mathrm{g} \log \mathrm{K}$ 
La constante d' es estimada por la fórmula:

(20) $d^{\prime}=\sum(\log X-f \log D-g \log K) / N=1.3700$

De la formula (20) podemos calcular las productivida des marginales:

(21) $\quad d x / d D=f \bar{x} / \bar{D}=3294,96$

(22) $d x / d K=g \bar{x} / \bar{K}=0,235206$

La interpretación de estos resultados ( computados para las medias de todas las variables ) es:

Si en Ecuador las condiciones son similares a las del perfodo investigado, y si ceteris paribus el empleo aumenta en un 0 brero, el producto va a aumentar en $3 \mathrm{mil}$ sucrès, aproximadamente.

Si ceteris paribus el stock de capital aumenta en un mi 116 n de sucres, el producto va a aumentar en 235 mil sucres, aproxi madamente.

Una modificacion importante puede ser introducida en la función de producción (5). Designamos por :

$$
\text { (23) } x_{t}=Y_{t} / N_{t} P_{t}
$$

el producto nacional real per cápital y también por:

$$
\text { (24) } e_{t}=D_{t} / N_{t}
$$

la proporción de la población total empleada, Nuestra función modificada de producción contiene tambien un trend exponencial ( lineal en lo 
garitmos) y puede ser representada como:

$$
x_{t}=A+B t-g \log N_{t}+f \log e_{t}+g \log K_{t}
$$

Los parámetros A y B son estimados con ayuda de los datos del perfodo $1950-61$, y el resultado final es:

(26) $\log x_{t}=1,32186+0,00739 t-0,5881 \log N_{t}+$

$$
0,4119 \log e_{t}+0,5881 \log K_{t}
$$

El coeficiente de regresión de $t(0,00739)$ corresponde como logaritmo al número 1, 017 y representa un aumento medio del trend exponencial de la producción de 1,7\% aproximadamente, 10 que puede explicarse por el prọgreso técnico.

4.

\section{Un modelo de largo plazo}

El modelo estático y el modelo dinámico están en términos monetarios. Pero, para un pals subdesarrollado como Ecuador un análisis de hechos reales es mucho más importante. Definimos todas nuestras variables en términos reales.

(a) Variables endogenas:

$\mathrm{x}=\mathrm{Y} / \mathrm{NP}=\mathrm{X} / \mathrm{N}$ P. I. B real per cápita

C - C/NP - consumo real per cápita

$\mathrm{W}=\mathrm{W} / \mathrm{P}$ salario real per cápita

e D $/ \mathrm{N}$ proporción de la población empleada

$k=K / N P$ capital real per cápita

(b) Variables ex6genas

i - I/NP inversión bruta real per cápita 
h - H/NP consumo dẹl gobierno, mâs expor taciones, menos importaciones reales per câpita.

Nuestro sistema consiste siempre de cuatro ecuaciones:

$$
c=a+b x-497,2667+0,5699 x
$$

Funcion del consumo:

(28) $\quad x=c+i+h$

Definición del producto real, per cápita:

(29) $f x=w . e$

Demanda de empleo. Consecuencia de la teorla de la pro ductividad marginal.
(30) $\log x=d^{\prime}+f \log e+g \log k=d^{\prime}+f \log e+$ g $\log \mathrm{i} /(1-\mathrm{A})=1,37541-0,5881 \log \mathrm{N}+0,4119$ $\log e+0,5881 \log i /(1-A)$

$\mathrm{Si}$

(31) $k_{t+1}=A k_{t}+i_{t}$

Función de produccion. La segunda forma de esta ecuación se explica por la relación (17), formación de capital a largo plazo. Este es un modelo de tipo keynesiano para predicción a largo plazo. El nivel de precios $\mathrm{P}$ no es ahora parte del modelo, perote nemos en su lugar los salarios definidos en términos reales $v$. Además, en lugar del empleo D tenemos la proporción del empleo en relación de la poblacion: e.

Es tambien interesante calcular las elasticidades parciales de todas las variables endogenas (c, $x, w, e)$ en relación con cada 
una de las variables exógenas $(i, h, N)$.

Denotamos las elasticidades parcial es como:

(32) $\quad$ E $y / E x=(d y / d z) .(z / y)$

Tenemos de las primeras dos ecuaciones de nuestro sistema (21) y (22):

(33) $E x / E i=i /(1-b) x=0,33$

(34) $E x / E h=h /(1-b) x \cdots-0,3316$

(35) $E_{c} / E_{i}=b i /(1-b) c=0,2629$

(36) $\mathrm{Ec} / \mathrm{Eh}=\mathrm{bh} /(1-\mathrm{b}) \mathrm{c}=0,2641$

Las elasticidades parciales de $\mathrm{x}$ (Producto Interno real per cápita) y c (consumo real per cápita) en relación a la población (N) son cero.

Usamos estos resultados y las dos ultimas ecuaciones de nuestro sistema (22) y (23) para el cálculo de las elasticidades parciales de $\mathrm{w}$ y de e:

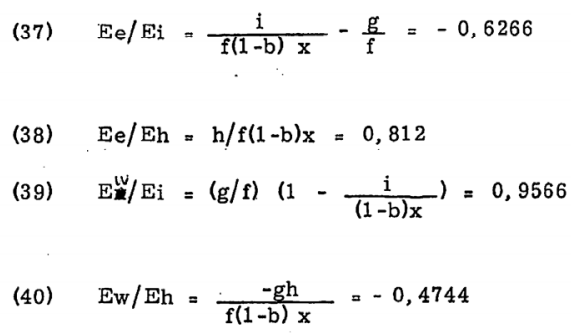

$\mathrm{La}$ interpretacion de estos resultados es: $\mathrm{Si}$ ceteris paribus la Inversión real per cápita aumenta en 1\%, el producto real per 
cápita aumenta en $1 / 3 \%$, si ceteris paribus la suma del consumo de gobierno más exportaciones menos importaciones (reales per cápita) aumenta en $1 \%$, el Producto real per câpita va a disminuir en $1 / 3 \%$, aproximadamente. Si ceteris paribus, uno $u$ otro, la inversion real per cápita o la suma de consumo del gobierno más exportaciones me nos importaciones (todos reales, per cápita) aumentan en $1 \%$, el con sumo real per cápita va a aumentar en $1 / 4 \%$, aproximadamente.

También, si ceteris paribus la inversión real per cápita aumenta en $1 \%$, la proporción de la población empleada va a dismi nuir en $0,6 \%$, si ceteris paribus la suma de consumo de gobierno más exportaciones me nos importaciones ( reales per cápita ) aumenta en $1 \%$, la proporción de la población empleada va a aumentar de $0,8 \%$.

Si ceteris paribus la inversión real per cápita aumen ta en $1 \%$, el salario real va a aumentar en $0,96 \%$, si ceteris paribus la suma de consumo de gobierno, más exportaciones menos importaciones ( reales per cápita) aumenta en $1 \%$ el salario real va a disminuir en $1 / 2 \%$, aproximadamente.

\section{Bibliografia}

B. von Hohenbalken y G. Tintner: Econometric models of the O. E. E. C. members, The United States and Canad, and Their application to economic policy. Weltwirtschaftliches Archiv, vol 89, 1962, pp. 29-86. L. Klein: a textbook of econometrics, Evaston III, 1953, pag. 191. 


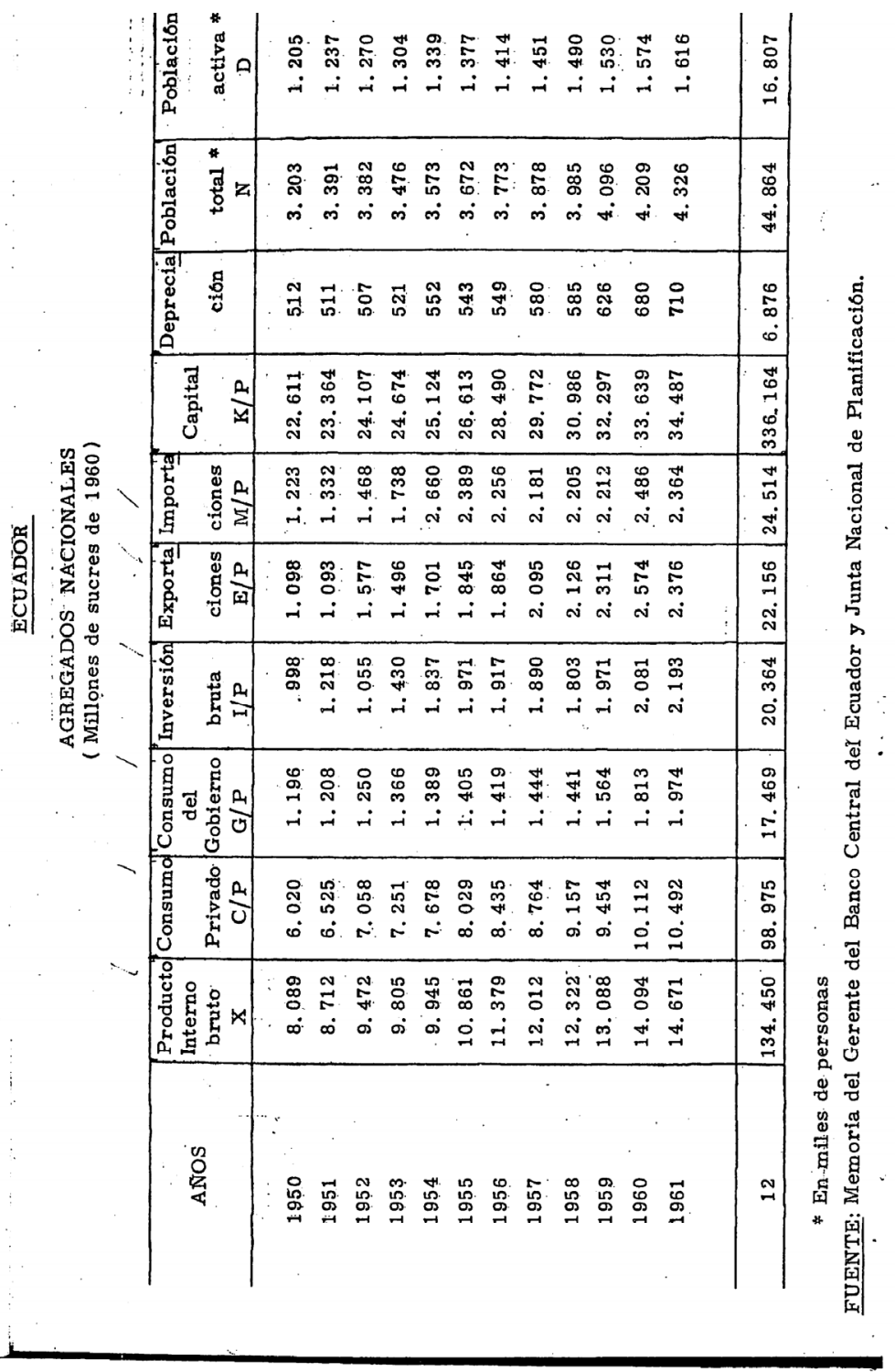




\section{BIBLIOGRAFIA}

Cohb Charles W. y Douglas Paul H. "A Theory of Production" (The American Economic Review"): "Un esquema completo para computar todas las elasticidades de demanda directa y cruzada en un modelo con muchos sectores", 1959, Econometrica, Vol. 18, No. 1, marzo, 1928-

Friedman, Thomas L."Gracias por llegar tarde". DEUSTO, Grupo Planeta, Colombia 2018.

Frisch, Ragnar. "Un esquema completo para computar todas las elasticidades de de.manda directa y cruzada en un modelo con muchos sectores", 1959, Econometrica

Frisch Ragnar "Nota sobre el Término'Econometria'", 1936, Econometrica .

Frisch, Ragnar: . "Un esquema completo para computar todas las elasticidades de demanda directa y cruzada en un modelo con muchos sectores", 1959, Econometric-

Haavelmo Trygve at the Cowles Commission. Article in Econometric Theory 31(01):84 - February2014DOI: 10.1017/S026646661400019.

Moncayo, Patricio." La planificación estatal en el interiuego entre desarrollo y democracia". Flacso, Ecuador, 2017 -

Salgado, Germániç, Edit. "25 años de planificación", Junta Nacional de Planificación. Quito, Ecuador, 1979-

Tinbergen, Jan. Econometrics . https://www.jstor.org/stable/2226757

Tintner Gerhard. "Methodology of mathematical economics and econometrics" Chicago : University of Chicago Press, 1968.113 p.

Tintner, Gerard, Econometrics. (London: Allen and Un- win, 1951. Pp. xii)

Tintner, Gerhrd\&Dávila Oswaldo, "Funciones de Producción para la Economia Ecuatoriana"- Junta Nacional de Planificación, Quito, Ecuador.1962 


\section{ANEXO 2. LA LEY DE MOORE A TRAVÉS DE LOS PROCEDIMIENTOS INTEL}

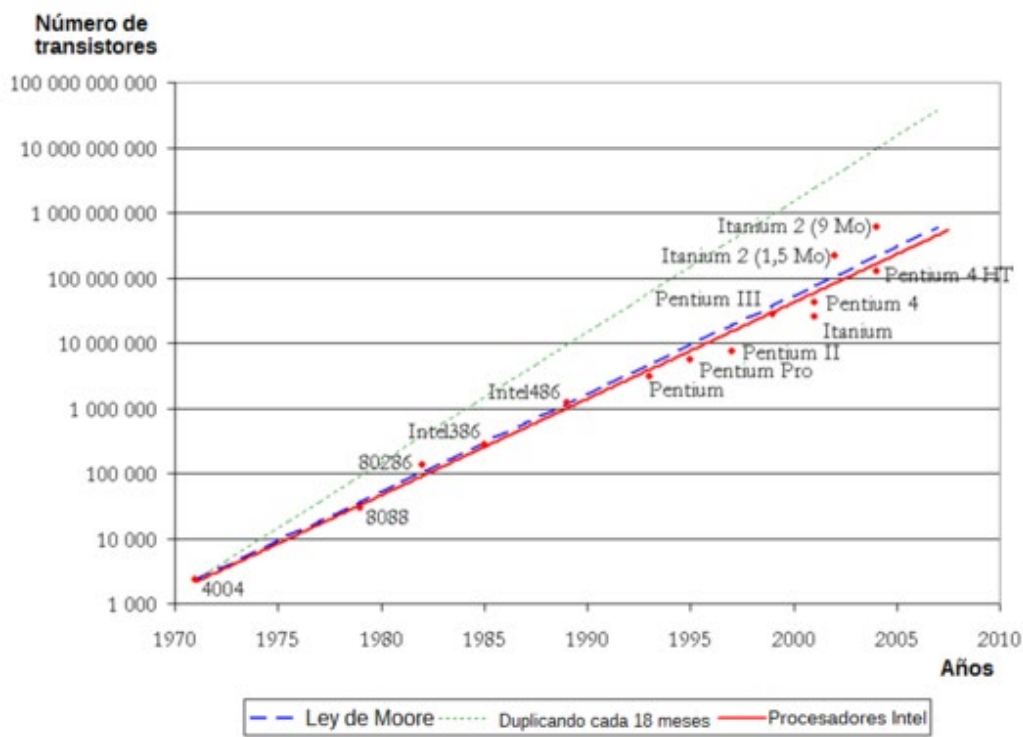

